\title{
Mathematical modeling radio tomographic ionospheric parameters reconstruction via nanosatellites constellation for conditions of incomplete source data
}

\author{
O.V. Phylonin ${ }^{1}$, P.N. Nikolaev ${ }^{1}$

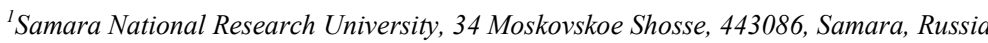

\begin{abstract}
The results of mathematical simulation of the formation of initial projection data for the problems of radio-tomography of the ionosphere using navigation satellites GPS and constellation of low earth orbit nanosatellites are presented. It is shown that for ring carriers (spherical layers), in the incompleteness of chordal data, the problems of reconstructing the electron density can be reduced to problems of low-angle tomography and the use of high-speed FBP algorithms.
\end{abstract}

Keywords: radio-tomography of the ionosphere; total electron content; nanosatellites; navigation satellites; FBP

\section{Introduction}

One of the promising areas of application of small satellites, including nanosatellites (NS), is their use for solving problems of radio-tomography, lidar-tomography of the ionosphere, analysis of the composition of cosmic radiation, etc. The radiotomography of the ionosphere (IRT) makes it possible to study various ionospheric structures, namely:

- ionization dips, in particular the total electron content (TEC);

- wave and quasiwave structure;

- traveling ionospheric disturbances (TIDs): "blobs", "patches", "bubbles", "ionization tongue";

- ionospheric "traces" of the corpuscular ionization, etc.

Studies of the structure of the ionosphere are important both for a theoretical understanding of the physics of processes occurring in it, and for many practical problems, since the ionosphere as a medium for the propagation of radio waves significantly affects the operation of various navigation, location and communication systems. The existing radar facilities and ionosondes allow only local diagnostics of the ionosphere. The creation of a fairly dense network of traditional ionosphere probes [1] is very difficult and expensive.

At the same time, existing satellite constellation such as:

- low earth orbit (LEO) constellation - Russian "cicada" American "Transit";

- high earth orbit (HEO) constellation GPS/GLONASS;

- network of ground-based receivers,

make it possible to sounded the ionosphere in different directions and to apply tomographic methods for reconstructing the ionosphere parameters.

In other words, IRT techniques allow to reconstruct the spatial structure of the electron density of the ionospheric plasma $[1,3]$. From the beginning of the 1990s, radio-tomography systems operate on the basis of LEO navigation systems. In recent years, radiotomographic studies have been actively carried out using data from HEO navigation systems [4, 5]. To identify different types of ionospheric radio tomography are used here, the terms LEO radio tomography, HEO radio tomography: LEORT and HEORT, respectively.

The radio-tomography of the ionosphere is based on the use, for example, of a two-frequency method, the essence of which can be clarified as follows. When the satellite is moving, ground receiving stations, or other satellites in the same orbit, continuous measurements of the phase delay of signals passing through the ionosphere at two frequencies are conducted. The initial data (chord data in the sense of Radon) are the corresponding phase paths of the radio signals measured in the lengths of the probing waves. If the frequency of the signals is much higher than some, the so-called plasma frequency, then from these data it is possible to determine the integral of the electron concentration along the trajectory of the satellite-receiver beam (the so-called total electronic content - TEC):

$$
\int_{l} N_{e}(r) d l=\left(\frac{L_{1}}{f_{1}}-\frac{L_{2}}{f_{2}}\right) \frac{f_{1}^{2} f_{2}^{2}}{f_{1}^{2}-f_{2}^{2}} \frac{c}{K}+\text { const }
$$

here: $c$ - speed of light, $K=40.308 \mathrm{~m}^{3} / \mathrm{s}^{2}$.

Thus, a typical problem of tomographic type arises - the definition of a function of several arguments with respect to a set of linear integrals from it (along the paths of the satellite-receiver). Essential features of this problem are:

- Firstly, the presence of an unknown phase constant for each beam of rays (since only phase change is possible for observations during the span of the satellite in the visibility zone).

- Secondly, the uncertainty of the problem due to the fact that only a small number of beams of satellite-receiver beams intersect the local area of space (and in the case of HEORT, there may also exist areas of total absence of data associated with the unevenness of the network of receiving stations). 
- Thirdly, when forming the initial chord data with the help of relatively HEO constellations of satellites, for example, GPS, forming highly stable radio pulses and low-orbiting groups of nanosatellites, recording the radio sounding signals, we deal with small amounts of chord data obtained in limited angles of convergence on a ring carrier.

The first problem is solved by using the phase difference approach (as an input difference of the integrals are taken over the neighboring rays, not the integrals themselves). To solve the second problem can be used iterative algorithms to ensure convergence to the normal solution (for a given norm), and also use special grid [6].

Regarding the third aspect it should be noted that the use of navigation satellite constellations such as GPS / GLONASS in combination with LEO nanosatellites constellation requires the development of innovative methods for the formation of the original projection data and algorithms for reconstruction of the desired spatial function distribution - such as electron density. This is due to the fact that the volumes and the possibility of such devices allow only transmit a digital code of the selected chord data in the Mission Control Center (MCC).

\section{Analysis of the possibilities of using satellite constellations GLONASS, GPS in the problems of radio-tomography of the ionosphere in combination with LEO clusters of nanosatellites}

Methods and means of HEORT, and LEORT allow to recover not only the natural ionospheric irregularities, but also to detect ionospheric disturbances generated by anthropogenic sources. In particular, perturbations caused by the rocket launches, industrial explosions, powerful HF radiation [5, 6]. Methods of RTI using GPS / GLONASS constellation and LEO (250 - 450) $\mathrm{km}$ NS clusters also enable to determine the plasma flows, considering the sequence of radio tomography section of the ionosphere.

Input data for ionospheric monitoring problems are measuring the radio signal phase (phase path) when passing them to the path from the satellite to the ground station receiver at two operating frequencies. For GPS systems, these frequencies are $f_{1}=1575.42 \mathrm{MHz}, f_{2}=1227.60 \mathrm{MHz}$. Radio signals are continuously emitted by satellite navigation systems; provide many opportunities for the implementation of the ionospheric plasma research using radio-tomography methods. At the same time, the use of LEO and HEO systems leads to two essentially different objectives. The classical methods LEORT let you receive the "instant" two-dimensional cross-section of the ionosphere with high resolution (20 - 30) km. Modern HEORT systems provide four-dimensional (spatial / temporal), the electron density distribution at a lower resolution (up to 30 - 50) km directly dependent on the density of the network of receiving stations in the region.

The next stage of the IRT is a process for sounding of the ionosphere with the help of signals emitted by navigation systems, and the registration of radio signals that have passed a certain layer of the ionosphere, by using LEO constellation of micro and nanosatellites (orbit altitude $220-270 \mathrm{~km}$ ). The satellites in the GLONASS system are moving in three orbital planes are separated relative to each other along the longitude of the ascending node $120^{\circ}$. The inclination of the orbital plane is $64.8^{\circ} \pm$ $0.3^{\circ}$. The orbits close to circular. The average height of orbits is $19,100 \mathrm{~km}$. Each orbital plane is uniformly 8 satellites. Satellite orbital period is 11 hours and 15 minutes $44 \pm 5$ seconds.

Satellites in the GPS system are moving in six orbital planes are separated relative to each other along the longitude of the ascending node at $60^{\circ}$. The inclination of the orbital plane is $55^{\circ}$. The orbits close to circular. The average height of the orbit $20189 \mathrm{~km}$. Four satellites located in each orbital plane. The orbital period of the satellite 11:00 $57 \mathrm{~min} 59.2 \mathrm{~s}$ (half of a sidereal day).

Each satellite has an atomic clock periodically synchronized by commands from Earth. Each satellite clocks synchronized satellite transmission via a special code signal. Before transmitting coded signals are modulated reports of movement trajectories of satellites and satellite parameters of time scales displacement models relative to the system scale. These messages are called navigation messages.

The structure of the signals transmitted by different satellites, such that:

- the receiver has the ability to separate these signals;

- assess their parameters;

- allocate navigation messages independently.

\section{Features of the formation of a LEO CubeSat cluster in relation to the satellite constellation (GLONASS, GPS) to ionospheric radio tomography study}

To solve the problems of IRT by using navigation satellite constellations (GLONASS, GPS) and LEO clustered systems of CubeSat format, it is obviously necessary to arrange the NS in this orbit so that the conditions for obtaining the initial projection data in the sense of Radon's inverse are satisfied. It is clear that in this sense, the locations of the navigation satellites are rigidly fixed, therefore, the formation of the initial projection data is possible only through the configuration of the orbital constellation of the NS. It should be noted that the IRT using small satellites constellation can be implemented in several ways:

1. By placing constellation of small satellites equipped with transmit-receive systems on the low and medium orbits [7]. Sounding is performed along the chord direction in a ring layer (2D reconstruction problem) using the transmitters and receivers installed on each small satellite. Reconstruction accuracy at radio-tomography such organization depends on the frequency stability of the transmitters emitted radio wave pulses, the accuracy in determining the location of the small satellite in orbit, of the orbit itself forms etc. Furthermore, the pulses emitted by each small satellite transmitter should be identical, since it also determines the accuracy of the reconstruction of the desired functional distributions. It is clear that it is possible to meet these conditions, which allow obtaining an acceptable accuracy of IRT reconstruction [8], only in devices of relatively large mass, 
since on their board it is necessary to mark highly stable transmitters, high-precision clocks, sensitive radio signal receivers, microprocessor control modules and preliminary data processing.

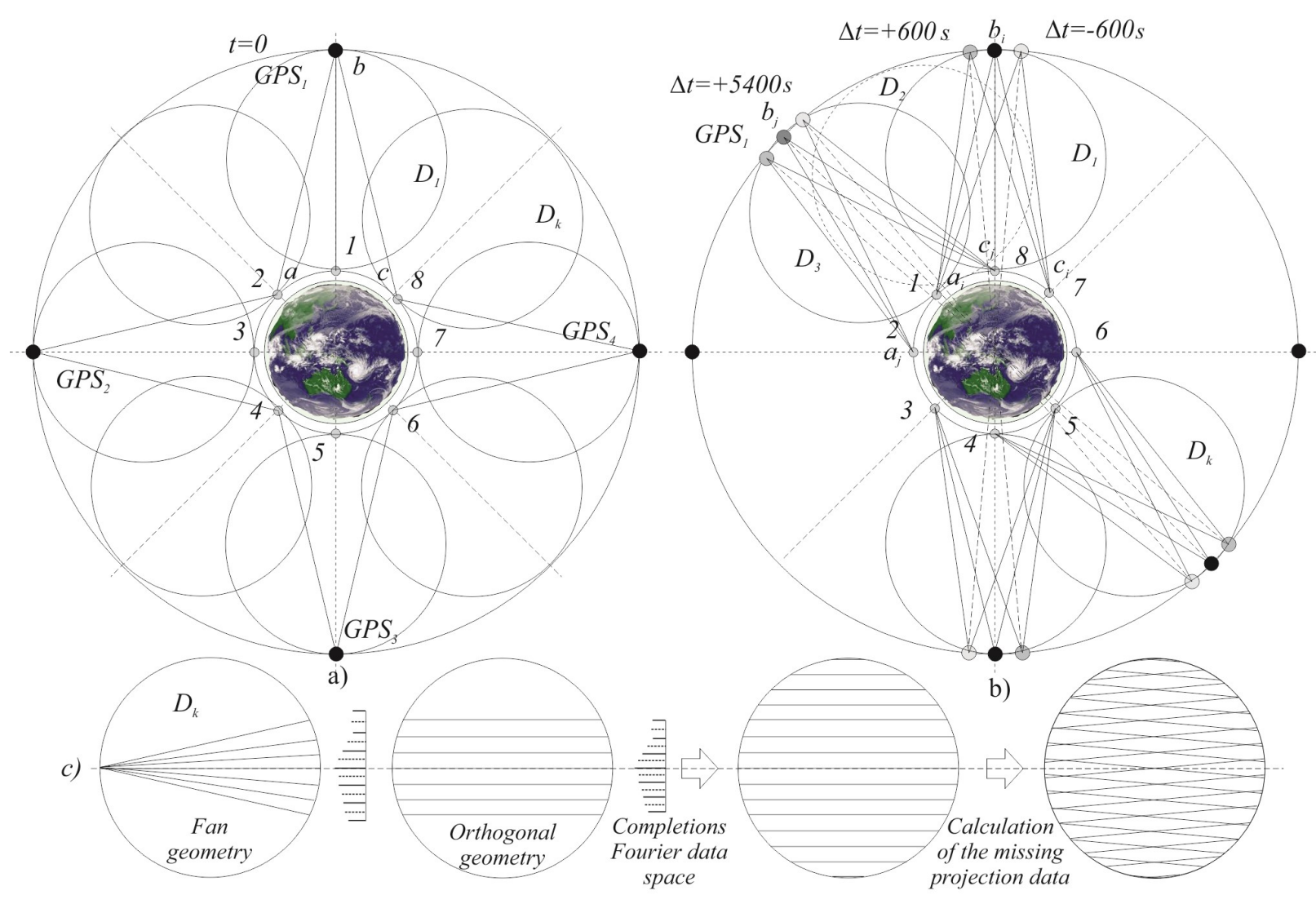

Fig. 1. Example of orbital configuration of GPS and LEO NS cluster for the IRT.

2. At that time, using radio signals of high quality of constellation of navigation satellites and a group of nanosatellites, a completely satisfactory solution of the problems of IRT is possible. Two frequency radio signal receivers, microprocessor control modules and preliminary processing of initial data, as well as transmitters of one-dimensional data sets to the control center, must be installed on board each NS.

Fig. 1 shows an example of the organization of an orbital constellation consisting of four GPS satellites (the average height of the orbit $20189 \mathrm{~km})$ and eight nanosatellites placed in low orbit $(220-270) \mathrm{km}$. The orbital plane, in this case the same - it is assumed the two-dimensional solution to the problem of IRT. Recall that the orbital period GPS - the satellite is 11 hours 57 minutes 59.2 seconds (half a sidereal day), and NS treatment period $(80 \div 90)$ minutes for definiteness choose 90 minutes. It is obvious that for half a sidereal day, each of the NS, who is in a low orbit, make eight orbit pass. At present, many researchers to solve the ionospheric radio tomography problem apply algebraic method of reconstruction of the desired functionality distributions, e.g., the electron density distribution [2]. Indeed, this approach makes it possible to get the maximum resolution and accuracy ionospheric radio tomography problems, but at the same time requires huge computational costs. Significantly lower amounts of computational procedures allow tomography methods based on algorithms of convolution (FBP) and Fourier transforms (FT) [9]. The accuracy and resolution of the reconstruction are quite satisfactory.

When navigation satellites and NS are moving in their orbits, projection data corresponding to a certain angle of convergencethe angle between projections is formed. In particular, after $\Delta t=600 \mathrm{c}$ time interval the satellite will take the position shown in Fig. 1 a), therefore, the convergence angle of will be $\Psi=2 \cdot( \pm 5)=10$. Due to the orbital motion of the satellite in a position $\Psi=+5$ to register the chord data will be NS by numbered $8,7,6$. Hence the issue for the microprocessor module processing raw data - the redistribution of integral chordates values of the on the corresponding recovery zone $D_{i}$. The signals from satellites are recorded respectively Ns triple numbered $2,3,4, \ldots, 6,7,8$, taking into account the orbital NS bias. Fig. 1 b) shows the geometry of chord formation data over a time interval $\Delta t=+5400 \mathrm{c}$.

Note that, as during orbital period navigation satellite, nanosatellites make eight orbit passes, it needs careful conversion of chord data for each of the reconstructed area $D_{i}$. The number of these circular areas should be more than twice as shown in Fig. 1 a).

Fig. 1 c) represented by the ideology of the pre-calculates base projection data to reconstruct the desired functionality distributions, for example, the TEC with the methods of few-view computer tomography. Its essence boils down to the following provisions:

- Implemented conversion of projection data from the fan-beam geometry to orthogonal geometry, but the reconstruction of the circular area is not completely filled with the chords of the projection; 
- On the basis of a priori data, using interpolation methods in Fourier space completions produced projection data (on circular harmonics) [9] - to completely fill the circular recovery zone;

- With the help of transmitters installed on each NS, are transmitted one-dimensional projection data in the MCC, and the corresponding received complete a definition data for each projection. The number of the actual results of the projections is not enough to reconstruct the size of the matrix $n \times n$.

- Taking into account the a priori data, using the properties of symmetry of the Fourier images, using multiprocessor computing complexes MCC made completions missing projections, so that the condition [9]: the number of projections, in all corners of the projection should be about 1.5 times greater than the dimension recovered format $n \times n$ (in one dimension - $n$ );

- The final stage of the procedure is performed each convolution kernel with low-frequency projection and rear projection the restoration of the desired functional distribution for each of the circular zone $D_{i}$. Then, using interpolation methods, recalculates the required data in the annular zone.

Thus, based on their characteristics produce raw projection data for IRT problems using constellation of navigation satellites and clusters of NS, it is easy to define the conditions to be met by hardware modules installed on each CubeSat:

- On each NS to be installed highly sensitive receivers for frequencies (for the reception of signals from GPS devices);

- Each NS should contain module to determine its location on the orbit by GPS data;

- Computational modules are installed on each NS must provide the required source data processing speed. Perform the appropriate procedures for conversion of projection data of a fan in an orthogonal geometry, redefine the number of chords to fill each round of reconstruction $D_{i}$. Achieving these goals is only possible when using multiprocessor computing modules equipped with the appropriate amount of RAM.

- To send the original one-dimensional projection array to the CPU required transmitters with a wide bandwidth. From the standpoint of reliability, each satellite must contain two such from each transmitter is capable of transmitting data, e.g., each pair of GPS.

- To coordinate referred modules each NS must contain the microprocessor control units.

- Each NS must be a certain way to orient in space and place on a circular orbit at equal distances from each other, for this purpose in each of the NS shall be provided accommodation compact three-axial gyroscope, and a set of actuators.

In order to accommodate the listed modules and accessories to the NS must use 7U CubeSat format. This format is an assembly CubeSat as a Makarov three-dimensional cross and contains $71 \mathrm{U}$ CubeSat format modules. This design allows you to install it on additional self-extracting solar panels, self-parabolic reflector antennas for receivers and transmitters of GPS signals to communicate with MCC. To increase the power available, the surface of 3D 7U CubeSat satellite is covered with solar panels. Conclusion and startup cluster consisting of those of the NS by means of "Soyuz", in which a transition compartment for up to 4 transport and display systems, each of which contains 4 3D 7U CubeSat format apparatus. The launch of clusters consisting of such NS can be carried out with the help of the Soyuz, in the transition compartment of which it is possible to arrange up to four deployment systems, each of which contains four devices of the 3D 7U CubeSat format.

\section{Mathematical model of radio tomography analysis of ionospheric parameters using GPS system and nanosatellites cluster}

The effectiveness of the proposed methods radio tomography of the ionosphere using navigation systems such as GPS / GLONASS and LEO NS clusters depends on many factors, such as a function of the gravitational field potential in the plane of the NS orbit, the curvature of the trajectory of the sounded antenna beam as a function of the refractive index, the functional variation of the amplitude, frequency, phase of the beam, etc. Take into account the impact of such factors on the adequacy of the tomographic reconstruction of ionosphere parameters of procedures you can use mathematical modeling techniques. In general, the mathematical model of the ionosphere parameters radio tomography analysis should take into account the following factors:

1. The direct and inverse problems of ionospheric radio sounding is necessary to determine the amplitude change, phase (frequency) radio waves to track the satellite - satellite. To this must be set the dependence of the refractive index of the height $n(h)$. This problem has been studied in detail by the authors [10]. The geometry of this problem is shown in Fig. 2 a). The points $L, G$ are located at altitudes $H_{l}, H_{g}$ of satellites. Earth Center designated $O$ point, in general, radial line $L T G$ in point $T$ passes at the minimum height above the Earth's surface $H$. The radial line at a higher level, in areas $L L_{1}$ and $G G_{1}-$ straight and in the field $L_{1} G_{1}$ because of the influence of media is rejected by the angle of refraction $\xi$. Assuming that the ionosphere is a local spherically symmetric environment can be negligible horizontal gradients of environment (near the point $T$ - line $L_{1} G_{1}$ ), and assume that the rate $n(r)$ depends only on the distance $O C=r=a+h$. Introduce: $h$ - the height of a point $a-$ the radius of the Earth; $\theta$ - a central angle, $g_{g}=a+H_{g}, r_{l}=a+H_{l}, r_{l}=a+H$ respectively, of the distance between $O G, O L$ and $O T$. For a spherically symmetric medium following formulas [11]:

$$
\begin{aligned}
& n(r) r \sin \gamma-\text { const, } \\
& P \Delta S-\text { const, }
\end{aligned}
$$


here $\gamma$ - the angle between $r$ and the unit vector radial lines $l_{0}$. Expression (2) determines the flux density in the cross section of the ray tube $\Delta S$, which makes it possible to calculate the change $P$ due to refraction. Since the refractive index differs only slightly from unity, it is customary [11] to use parameter $N=n-1$ which depends on the pressure $P_{a}$, temperature $T$ and humidity $w_{a}$ as follows:

$$
N=\frac{77.6}{T}\left(P_{a}+\frac{481 w_{a}}{T}\right) \cdot 10^{-6} .
$$
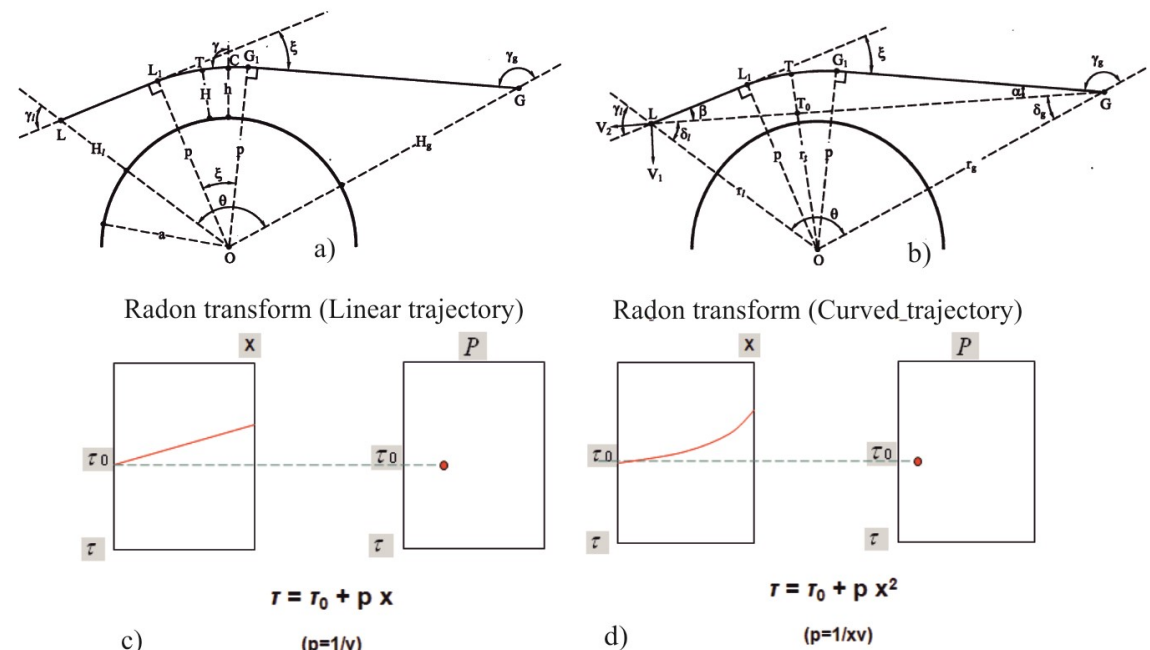

c)

$(p=1 / v)$

d)

Fig. 2. Illustrate the effect of the ionospheric refractive index of the sounding antenna beam and the Radon image from sounding geometry.

In this model, the height profile of the given refractive index can be approximated by:

$$
N(h) \approx N_{0} \exp \left(-b_{l} h\right)
$$

when:

$$
b_{l}=0.1 \cdot \ln \left(\frac{9.2 \cdot 10^{-5}}{N_{0}}\right) .
$$

Since the real profile $N(h)$ differs from (4), it can be used as an approximation:

$$
N(h)=N_{0} \exp \left(-a_{1} h^{2}-b_{1} h\right) .
$$

there $a_{1}, b_{1}-$ const [12].

The above plasma refractive index, for high frequencies:

$$
N_{p}(h)=-\chi N_{e} f^{-2}, \quad \chi=40.4(S I) .
$$

For the upper part of the ionosphere $N_{e}=N_{m} \exp \left[-b_{2}\left(h-h_{m}\right)\right]$, here $N_{m}$ - the electron density in the main ionospheric maximum at altitude $h_{m}$. Below $h_{m}$, you can use the approximation:

$$
N_{e}(h) \approx N_{m}\left[1-\left(\frac{h_{m}-h}{c_{2}}\right)^{2}\right],
$$

Where $c_{2}$ - the nominal thickness of the lower part of the ionosphere.

The paper [11] shown that for beam line in a spherically symmetric medium satisfies the relation:

$$
\operatorname{tg} \gamma=\frac{p}{\left(r^{2} n^{2}(r)-p^{2}\right)^{1 / 2}}
$$

which implies that the radial line defined by the altitude $n(h)$ profile and the parameter $p$.

The radius of curvature of the beam in a spherically symmetric medium:

$$
R_{0}=\frac{a+h}{\sin \gamma+(a+h) \frac{d \gamma}{d h} \cos \gamma} .
$$

Refraction angle:

$$
\xi=2 \int_{H}^{\infty}\left(\frac{d \gamma}{d h}+\frac{d \theta}{d h}\right) d h, \rightarrow \xi=-2 \int_{H}^{\infty} \frac{1}{n} \frac{d n}{d h} \operatorname{tg} \gamma d h .
$$


For model experiments it is advisable to use the approximation:

$$
\xi=N_{0}(2 \pi b a)^{1 / 2} \exp (-b H)
$$

Note: In the atmosphere of refraction does not depend on the wavelength and angle $\xi$ in the ionosphere proportional to the square of the wavelength. The vertical gradient of the electron density:

$$
\frac{d n}{d h}=-\chi f^{-2} \frac{d N_{e}}{d h}
$$

The above expressions for the refractive index, the radius of curvature, and the refraction angle make it possible to simulate in detail the ray lines along satellite-satellite lines. Use as a projection data values integrated radio signal intensity along a curved radial lines, including in cases where NS is provided with receivers are on the horizon line of sight (see the eclipsing of reference. Fig. 2 c) and d)).

2. Refraction attenuation, frequency and phase changes sounding radio waves make it possible within the framework of the model of the ionosphere sounding radio tomography obtain more accurate data about Radon icons is formed in the projection data generated. This, in turn, all other things being equal, allows a high degree of precision to carry out reconstruction of the desired functional distributions. Consider ray tube at the point $G$ (see. Fig. 2 b)) with an angular size $d \gamma_{g}$ in the perpendicular plane of its size $d \chi$, and calculate its size at the point $L$. From the above geometry seen that $L L_{2}=r_{1} d \theta$ the linear dimension of the ray tube at a point $L$ equal $L L_{3}=r_{1} \cos \gamma_{1} d \theta$ to one can show that the cross-sectional area at the point $L$ is:

$$
S_{1}=r_{1}^{2} \sin \theta \cos \gamma_{1} d \theta d \chi
$$

In the absence of refraction ray tube would have a cross-section in the region of point $L$ :

$$
S_{o}=L^{2} \sin \gamma_{g} d \gamma_{g} d \chi
$$

here $L=\sqrt{r_{l}^{2}+r_{g}^{2}-2 r_{l} r_{g} \cos \theta}$ - the distance between points $L, G$. Define refractive weakening as the ratio of the power flux density in the presence of refraction $P_{l}$ and its absence $P_{0}$ :

$$
X=\frac{P_{l}}{P_{0}}=\frac{S_{0}}{S_{l}}=\frac{L^{2} \sin \gamma_{g} d \gamma_{g}}{r_{1}^{2} \sin \theta \cos \gamma_{l} d \theta} .
$$

Authors [11] lead (15) to mean:

$$
X=\frac{p\left(r_{l}^{2}+r_{g}^{2}-2 r_{l} r_{g} \cos \theta\right)}{r_{l} r_{g} \sin \theta\left[\left(r_{l}^{2}-p^{2}\right)^{1 / 2}+\left(r_{g}^{2}-p^{2}\right)^{1 / 2}-\frac{d \xi}{d p}\left(r_{l}^{2}-p^{2}\right)^{1 / 2}\left(r_{g}^{2}-p^{2}\right)^{1 / 2}\right]} .
$$

In (16) it is assumed that the radio sounding $n_{g}=n_{l}=1$. For small angles of refraction at high frequencies, the sounded beam:

$$
X=\frac{p\left(L_{g}+L_{l}\right)^{2}}{r_{g} r_{l} \sin \theta\left(L_{g}+L_{l}+L_{g} L_{l} \frac{d \xi}{d p}\right)} .
$$

From (17) it follows that in the case of sounding system consisting of HEO - LEO satellites $L_{g}>L_{l}$ :

$$
X \approx \frac{p}{r_{l} \sin \theta\left(1+L_{l} \frac{d \xi}{d p}\right)} .
$$

For small angles of refraction $p \approx r_{l} \sin \theta$ that occurs for the chord data in the center of the fan-expression (18) sensing beam can be written as:

$$
X \approx\left(1-L_{l} \frac{d \xi}{d p}\right)^{-1}
$$

In the exponential approximation $N(h)$ we can use the ratio:

$$
X \approx\left[1+b L_{l}(2 \pi b a)^{1 / 2} N_{0} \exp (-b H)\right]^{-1} .
$$

Doppler frequency change in the ionosphere $\Delta f_{S}$ : 


$$
\Delta f_{S}=\lambda^{-1}\left(V_{2} \cos \beta+V_{1} \sin \beta\right)
$$

where $V_{1}, V_{2}$ - on the satellites velocity projection beam line at the point $L$. If there is no change in the frequency of the ionosphere due to the satellite motion is:

$$
\Delta f_{0}=\lambda^{-1} V_{2} \text {. }
$$

Thus, the change in frequency only through the ionosphere:

$$
\Delta f=\Delta f_{S}-\Delta f_{0}=\lambda^{-1}\left[V_{2}(\cos \beta-1)+V_{1} \sin \beta\right]
$$

That is, ionospheric frequency change depends from the angle $\beta$ and velocity of the satellite components $V_{1}, V_{2}$. For small angles of refraction and $r_{g}>>r_{l}$ can be used:

$$
\Delta f \approx \lambda^{-1} V_{1} \xi
$$

To account for the phase change of the probe beam $\Delta \varphi=\varphi-\varphi_{0}$, where $\varphi_{0}$ - the phase for the curved path - phase for the rectilinear propagation of the beam, i.e., when the ionosphere is absent in this model, the following relationships are used:

$$
\varphi=\frac{2 \pi}{\lambda} \int_{G}^{L} n d l=\frac{2 \pi}{\lambda}\left(\int_{r_{l}}^{r_{g}} \frac{n^{2} r d r}{\left(n^{2} r^{2}-p^{2}\right)^{1 / 2}}+\int_{r_{l}}^{\eta} \frac{n^{2} r d r}{\left(n^{2} r^{2}-p^{2}\right)^{1 / 2}}\right), \varphi_{0}=\frac{2 \pi}{\lambda}\left[\left(r_{g}{ }^{2}-p_{0}^{2}\right)^{1 / 2}+\left(r_{l}^{2}-p_{0}^{2}\right)^{1 / 2}\right],
$$

here $p_{0}$ - the minimum distance from the line $G L$ to the center of the Earth. Importantly, ionospheric value $\Delta \varphi$ proportional to the wavelength, and the refractive attenuation $X$, frequency change $\Delta f$ and phase $\Delta \varphi$ can be expressed in terms of the angle of refraction, so depending $X(H), \Delta f(H), \Delta \varphi(H)$ interconnected. This approximate relationship, provided that $V_{1}$ it is not time-dependent, is given [11]:

$$
\begin{aligned}
& X=\left[1-\left(\frac{L_{l} c}{f V_{1}^{2}}\right) \cdot \frac{d(\Delta f)}{d t}\right]^{-1}, \\
& X=\left[1-\left(\frac{L_{l} c}{2 \pi f V_{1}^{2}}\right) \cdot \frac{d^{2}(\Delta \varphi)}{d t^{2}}\right]^{-1}
\end{aligned}
$$

Note: If the solution of inverse problems of monitoring ionospheric parameters from the experimentally obtained dependences $\Delta \varphi(t), \Delta f(t), X(t)$ is necessary to reconstruct the profiles $N(t), N_{e}(t)$, in such cases, the satellite coordinates and their speed must be known.

3. The greatest difficulty in solving inverse problems of monitoring ionospheric parameters using navigation satellites sources of sounding signals and LEO NS clusters - sounding recording radio pulses is the problem of reconstruction of the desired functional distributions $N(r, t), N_{e}(r, t)$ in sensing zone. Recall that the sensing zone for 2D problem is an annular carrier (in the form of a spherical layer for applications of direct 3D - reconstruction), determined by the diameter and the orbits of the navigation satellites and NS clusters. To reconstruct the desired functional $N_{e}(r, t)$ type distributions by fast algorithms based on the Radon transform must be reformulated Radon's theorem for the carrier ring, or to fill a circular annulus reconstruction carriers $D_{i}$, as it is shown in Fig. 2. In the first case will have to deal with very large data sets while the final resolution in the reconstructed image will be low, of the order $(200 \times 200) \mathrm{km}$, which is much lower than with traditional methods tomography [1]. The second case is for these preferred methods, despite the fact that there is a problem in calculation of the probing beam parameters at the boundaries of each circular zone. Recall that for the inverse Radon transform function $N_{e}(r, t)$ at any given time $t$ can be written as:

$$
[R]^{-1} N_{e}(r, \varphi)=\frac{1}{2 \pi^{2}} \int_{0}^{\pi} \int_{-\infty}^{+\infty} \frac{1}{r \cos (\theta-\varphi)-l} \frac{\partial N_{e}(l, \theta)}{\partial l} d l d \theta
$$

Represent the action of the operator $[R]^{-1}$ in the form of a sequence of simpler operators $R^{-1}=B H_{l} D_{l}$. The integral in equation (27) is improper, since $r \cos (\theta-\varphi)=l$ it diverges. It can be calculated by converting the integral in the sense of the Cauchy principal value, after entering the designation $r \cos (\theta-\varphi)=\tau$ :

$$
\left[H_{l}\left(N_{e l}\right)\right](l, \theta)=-\frac{1}{\pi} \lim _{\varepsilon \rightarrow 0}\left\{\int_{-\infty}^{l-\varepsilon} \frac{N_{e}{ }^{\prime}{ }_{\tau}(\tau, \theta)}{l-\tau} \mathrm{d} \tau+\int_{1+\varepsilon}^{+\infty} \frac{N_{e}{ }^{\prime}{ }_{\tau}(\tau, \theta)}{l-\tau} \mathrm{d} \tau\right\}
$$


Calculate the integral can, if present in the Hilbert transform (28) in the form of a convolution of two functions $N_{e}{ }^{\prime} \tau(\tau, \theta)$ and $\chi(l)=-\frac{1}{\pi l}$, i.e., written in the form of relation:

$$
\left[H_{l}\left(N_{e^{\prime} l}\right)\right](l, \theta)=\left[N_{e}{ }^{\prime} * \chi\right]_{l}(l, \theta) .
$$

Run directly to the operation (29) cannot be performed, so we approximate the function $\chi(l)$ by a function $\chi_{A}(l)$, so that the following condition:

$$
\lim \left[N_{e}^{1} * \chi_{A}\right]_{l}(l, \theta)=\left[H_{l} N_{e l}^{1}\right](l, \theta)
$$

This approach, defined by (30), as is known [9] is called regularization method and the functions set $\{\chi(l) / A>0\}$ - is called a family of regularizing functions. As it is known the integral convolution of two functions can be replaced by multiplication of their Fourier spectra in the frequency domain, i.e.:

$$
\left[N_{e}{ }^{\prime} * \chi\right]_{l}(l, \theta)=F_{\omega_{l}}^{-1}\left[\left[F_{l} N_{e l}^{1}\right]\left(\omega_{l}\right) \cdot\left[F_{l} \chi\right]\left(\omega_{l}\right)\right],
$$

here:

$$
\left[F_{l}\left(N_{e^{\prime} l}\right)\right]\left(\omega_{l}\right)=\int_{-\infty}^{+\infty} N_{e}{ }^{\prime} l(l, \theta) e^{-i 2 \pi \omega_{l} l} d l,\left[F_{l} \chi\right]\left(\omega_{l}\right)=\int_{-\infty}^{+\infty} \chi(l) e^{-i 2 \pi \omega_{l} l} d l .
$$

A direct calculation of (31) is difficult, as the final function $N_{e l}(l, \theta)$ has an infinite range. A simple "truncation spectrum" is unacceptable, because it leads to the generation of noise due to the Gibbs phenomenon. In such cases, it decided to do, taking into account (30), as follows:

$$
\chi_{A}(l)=\int_{-\frac{A}{2}}^{\frac{A}{2}}[F \chi]\left(\omega_{l}\right) \cdot W\left(\omega_{l}\right) e^{2 \pi i \omega_{l} l} d \omega .
$$

Window function $W\left(\omega_{l}\right)$ must satisfy the following conditions:

$$
\begin{aligned}
& \qquad W_{\left(\omega_{l}=0\right)}=1 ; W_{\left(\omega_{l}\right)}=0 ;|\omega| \geq \frac{A}{2} \lim _{A \rightarrow \infty} W_{\left(\omega_{l}\right)}=1 ; W_{\left(\omega_{l}\right)} 0 \leq \omega_{l} \leq \frac{A}{2} ; \lim _{A \rightarrow \infty} \omega(l)=0, \rightarrow \omega(l)=\frac{1}{2 \pi} \int_{-\frac{A}{2}}^{\frac{A}{2}} W\left(\omega_{l}\right) e^{2 \pi i \omega_{l} l} d \omega_{l} \\
& \text { Fourier spectrum of the function } \chi(l) \text { is obvious: }
\end{aligned}
$$

$$
\left[F_{l} \chi\right]\left(\omega_{l}\right)=-\frac{2}{\pi} \int_{0}^{+\infty} \frac{\sin 2 \pi \omega_{l} l}{l} d l=-\operatorname{sgn}\left(\omega_{l}\right)
$$

in view of

$$
\omega(l)=\frac{1}{2 \pi} \int_{-\frac{A}{2}}^{\frac{A}{2}} W\left(\omega_{l}\right) e^{2 \pi i \omega_{l} l} d \omega_{l} .
$$

Approximating function $\chi_{A}(l)$ can be represented as:

$$
\chi_{A}(l)=-2 \int_{0}^{\frac{A}{2}} W\left(\omega_{l}\right) \sin \left(2 \pi \omega_{l} l\right) d \omega_{l} .
$$

If we calculate the limit of approximating function (34), i.e.:

$$
\lim _{A \rightarrow \infty} \chi_{A}(l)=\lim \left\{\frac{1}{\pi l}\left[W\left(\omega_{l}\right) \cos \left(2 \pi \omega_{l} l\right)-\int_{0}^{\frac{A}{2}} W^{1}\left(\omega_{l}\right) \cos \left(2 \pi \omega_{l} l\right) d \omega_{l}\right]\left(_{0}^{\frac{A}{2}}\right\}=-\frac{1}{\pi l},\right.
$$

it becomes apparent that the condition (30) holds, i.e.:

$$
\left[N_{e}{ }^{\prime} l^{\prime} * \chi_{A}\right]_{l}(l, \theta)=\int_{-\infty}^{+\infty} N_{e}^{\prime}{ }_{\tau}(\tau, \theta) \chi(l-\tau) d \tau .
$$

Note that the desired function $N_{e}(r)$ is defined in a finite region - property section is said to be defined on a finite medium, therefore, the area of its existence can be set in a range bounded by a circle of radius $R$, i.e.: $x^{2}+y^{2}=R^{2} ; \quad \rho_{l, \theta}=0 ; \quad|l| \geq R$. With this in mind, we integrate the right side of (40) by parts:

$$
\left[N_{e}{ }^{\prime} l^{\prime} * \chi_{A}\right]_{l}(l, \theta)=\int_{-\infty}^{+\infty} N_{e \theta}(\tau) \chi_{A}^{\prime}(l-\tau) d \tau,
$$

We now calculate the derivative of the function $\chi_{A}$ under the integral sign in (41), i.e.:

$$
\chi_{A}^{\prime}(l)=-4 \pi \int_{0}^{\frac{A}{2}} \omega W\left(\omega_{l}\right) \cos \left(2 \pi \omega_{l} l\right) d \omega_{l} .
$$


We introduce the notation $h(l)=\chi^{\prime}{ }_{A}(l)$, then we can write:

$$
N_{e}(l, \theta)=\left[N_{e} * h\right]_{l}(l, \theta) .
$$

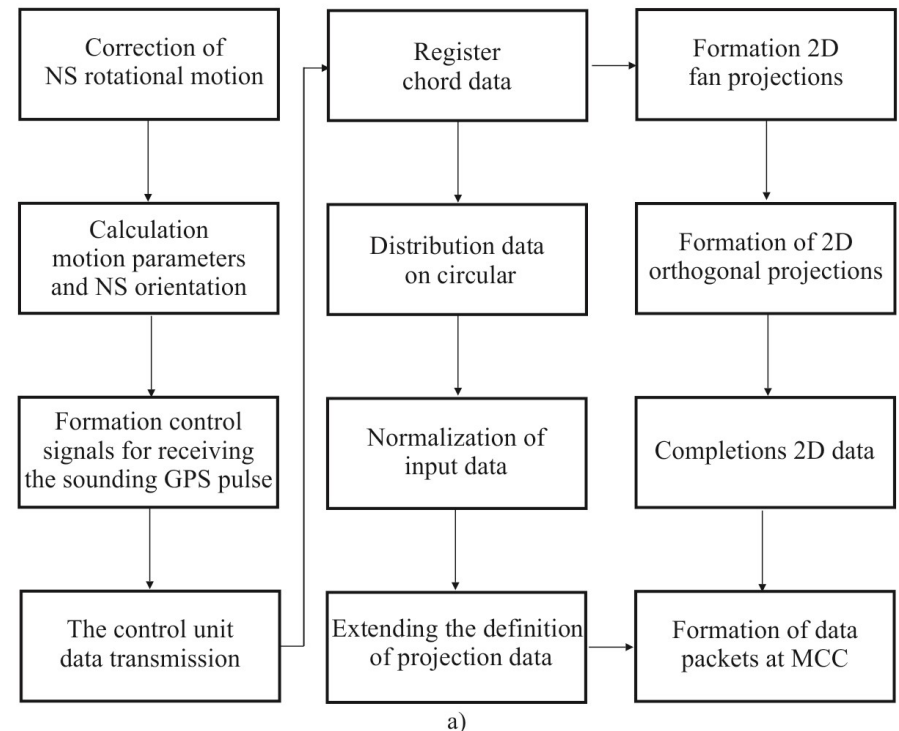

a)

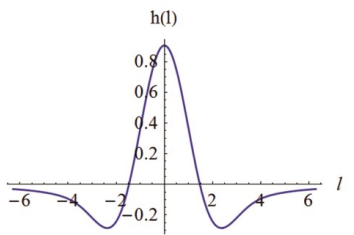

b)

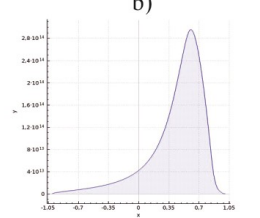

c)

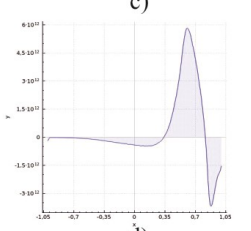

d)
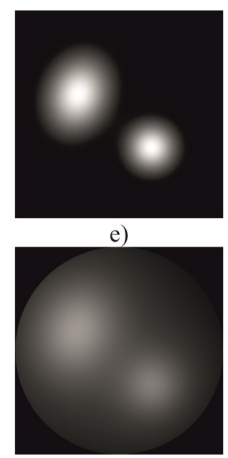

f)

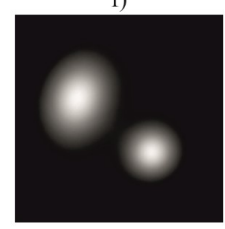

g)

Fig. 3. Illustrations of the stages of mathematical modeling procedures for solving the reconstruction inverse problem of distributions $N_{e}(r)$ when used GPS and NS cluster for radio sounding.

Formula (39) in accordance with expression (29) is an approximation to the Hilbert transform provided $A \rightarrow \infty$. We use the inverse projection operator, and write:

$$
N_{e}(r, \varphi)=\left[B\left(N_{e}\right)\right](r, \varphi)=-\frac{1}{2 \pi} \int_{0}^{\pi} N_{e}(r \cos (\theta-\varphi), \theta) d \theta
$$

Thus, we illustrate one possible way of approximating the inverse Radon transform, which reduces to two procedures: 1) projection function is convolve with the function $h(l)$ of certain expressions (39) and (40) respectively. 2) Perform the backprojection procedure.

Note: The selection of the appropriate "window" is usually performed fairly subjectively, based on a visual assessment of the quality of the reconstruct image. In this case, a compromise between resolution and noise level is usually sought.

For the purposes of IRT the authors developed low-frequency "window" for fast convolution algorithm applied to the methods of radio sounding using satellite clusters, which are described in detail in $[13,14,15]$.

To study the possibilities of using NS clusters capable of detecting the sounding pulses from GPS navigation satellites for solving tomographic problems for reconstructing the desired functional distributions, for example, electron density, the authors carried out a full cycle of mathematical modeling of tomographic reconstruction procedures taking into account the factors considered above. The results are shown in Fig. 3.

Fig. 3 a) shows a block diagram of the control program modules and data pre-processing for the microprocessor-based systems installed on the NS. Low-frequency core view is shown in Fig. 3 b), Fig. 3c) and d) respectively show the generated projection range for the result of convolution of the projection to the core. Fig. $3 \mathrm{e}$ ) shows the model function, and Fig. $3 \mathrm{f}$ ) and g) of reconstruction by using different cores.

\section{Conclusion}

Creating a mathematical model of ionospheric parameters radio tomography procedures using navigation systems types GPS / GLONASS satellites used as sources of sounding signals and NS cluster, whose satellites are equipped with highly sensitive, stable receivers showed that this approach is a highly effective method of ionospheric research. Method of analysis of ionospheric, allowing near real-time analysis of the distribution function, e.g., the electron density, was proposed by authors. This method is relatively simple to implement, a methodological error of reconstruction by four NS constellation is (15 - 20) \% and can be reduced by increasing the number of the NS in the cluster.

\section{References}

[1] Kunitsyn V, Tereshchenko ED, Andreeva ES. Radio-tomography of the ionosphere. Moscow: fizmatlit, 2007.

[2] Andreeva ES, Galinov AV, Kunitsyn VE. Tomographic reconstruction of the ionospheric ionization ionization failure. Pis'ma v ZhETF 1990; 52(3): 783785.

[3] Bust G, Mitchell CH. History, current state, and future directions of ionospheric imaging. Reviews of Geophysics, 2008 ; 46 RG1003:.1-23.

[4] Nesterov IA, Kunitsyn VE. GNSS radio tomography of the ionosphere: the problem with essentially incomplete data. Adv. Space Res. 2011 ; 47: 1789-1803. 
[5] Kunitsyn VE, Nesterov IA, Padokhin AM, Tumanova Yu S. Radio-tomography of the ionosphere on the basis of GPS / GLONASS navigation systems. Radiotekhnika i elektronika 2011; 56(11): 1285-1297.

[6] Kunitsyn VE, Tereshchenko ED, Andreeva ES, Nesterov IA. Satellite radio sounding and radio-tomography of the ionosphere. Uspekhi fizicheskikh nauk 2010; 180(5): 548-553.

[7] Phylonin OV, Talyzin YuB. Mathematical modeling of the processes of studying planetary atmospheres with the help of colonies of small satellites. Proceedings III All-Russia scientific and technical conference "Actual problems of rocket and space technology". Samara, 2013; 245-248.

[8] Phylonin OV. Inverse ill-posed problems in space research. Samara, 2014; 478 p.

[9] Phylonin OV. Low-angle reconstructive tomography in a physical experiment. Saarbrucken, Germany: Palmarium Academic Publishing, 2012 ; 606 p.

[10] Kalashnikov IE, Matyugov SS, Yakovlev OI. Influence of the ionosphere on the parameters of the signal in the radio decompounding of the Earth's atmosphere. Radiotekhnika i elektronika 1986; 31(1): 56.

[11] Yakovlev O, Pavel'ev A, Matyugov S. Satellite monitoring of the Earth: Radiospheric monitoring of the atmosphere and ionosphere. M.: Knizhnyi dom LIBROKOM, 2010; 208 p.

[12] Bilitza D, McKinnell L-A, Reinisch B, Fuller-Rowell T. The International Reference Ionosphere (IRI) today and in the future. Journal of Geodesy 2011; 85: 909-920.

[13] Phylonin OV, Nikolaev PN. Monitoring of the state of the earth's ionosphere by a group of small satellites. Vestnik Samarskogo universiteta. Aerokosmicheskaya tekhnika, tekhnologii i mashinostroenie 2016; 15(1): 132-138.

[14] Phylonin OV, Belokonov IV, Nikolayev PN. Mathematical Modeling of Radio Tomographic Ionospheres Monitoring Via Satellite Constellation. Scientific and Technological Experiments on Automatic Space Vehicles and Small Satellites. Procedia Engineering 2015; 104: $131-138$.

[15] Phylonin OV, Belokonov IV. Investigation of the possibilities of spatial reconstruction of the parameters of the electronic component of the ionosphere using navigation satellites. Izvestiya of the Samara Scientific Center of the Russian Academy of Sciences 2014; 16(4-1): 47-53. 